\title{
Educational attainment impacts drinking behaviors and risk for alcohol dependence: results from a two-sample Mendelian randomization study with $\sim 780,000$ participants
}

\author{
Daniel B. Rosoff ${ }^{1} \cdot$ Toni-Kim Clarke $^{2} \cdot$ Mark J. Adams $\mathbb{1}^{2} \cdot$ Andrew M. McIntosh $\mathbb{D}^{2} \cdot$ George Davey Smith $\mathbb{D}^{3}$. \\ Jeesun Jung ${ }^{1} \cdot$ Falk W. Lohoff ${ }^{1}$
}

Received: 15 April 2019 / Revised: 5 September 2019 / Accepted: 20 September 2019 / Published online: 25 October 2019

This is a U.S. government work and not under copyright protection in the U.S.; foreign copyright protection may apply 2019. This article is published with open access.

\begin{abstract}
Observational studies suggest that lower educational attainment (EA) may be associated with risky alcohol use behaviors; however, these findings may be biased by confounding and reverse causality. We performed two-sample Mendelian randomization (MR) using summary statistics from recent genome-wide association studies (GWAS) with $>780,000$ participants to assess the causal effects of EA on alcohol use behaviors and alcohol dependence (AD). Fifty-three independent genome-wide significant SNPs previously associated with EA were tested for association with alcohol use behaviors. We show that while genetic instruments associated with increased EA are not associated with total amount of weekly drinks, they are associated with reduced frequency of binge drinking $\geq 6$ drinks $\left(\beta_{\mathrm{IVW}}=-0.198,95 \% \mathrm{CI}\right.$, -0.297 to $\left.-0.099, P_{\mathrm{IVW}}=9.14 \times 10^{-5}\right)$, reduced total drinks consumed per drinking day $\left(\beta_{\mathrm{IVW}}=-0.207,95 \%\right.$ CI, -0.293 to $\left.-0.120, P_{\mathrm{IVW}}=2.87 \times 10^{-6}\right)$, as well as lower weekly distilled spirits intake $\left(\beta_{\mathrm{IVW}}=-0.148,95 \% \mathrm{CI}\right.$, -0.188 to $\left.-0.107, P_{\mathrm{IVW}}=6.24 \times 10^{-13}\right)$. Conversely, genetic instruments for increased EA were associated with increased alcohol intake frequency $\left(\beta_{\mathrm{IVW}}=0.331,95 \% \mathrm{CI}, 0.267-0.396, P_{\mathrm{IVW}}=4.62 \times 10^{-24}\right)$, and increased weekly white wine $\left(\beta_{\mathrm{IVW}}=0.199,95 \% \mathrm{CI}, 0.159-0.238, P_{\mathrm{IVW}}=7.96 \times 10^{-23}\right)$ and red wine intake $\left(\beta_{\mathrm{IVW}}=0.204,95 \% \mathrm{CI}, 0.161-0.248\right.$, $\left.P_{\mathrm{IVW}}=6.67 \times 10^{-20}\right)$. Genetic instruments associated with increased EA reduced $\mathrm{AD}$ risk: an additional 3.61 years schooling reduced the risk by $\sim 50 \%\left(\mathrm{OR}_{\mathrm{IVW}}=0.508,95 \% \mathrm{CI}, 0.315-0.819, P_{\mathrm{IVW}}=5.52 \times 10^{-3}\right)$. Consistency of results across complementary MR methods accommodating different assumptions about genetic pleiotropy strengthened causal inference. Our findings suggest EA may have important effects on alcohol consumption patterns and may provide potential mechanisms explaining reported associations between EA and adverse health outcomes.
\end{abstract}

Supplementary information The online version of this article (https:// doi.org/10.1038/s41380-019-0535-9) contains supplementary material, which is available to authorized users.

$\triangle$ Falk W. Lohoff

falk.lohoff@nih.gov

1 Section on Clinical Genomics and Experimental Therapeutics, National Institute on Alcohol Abuse and Alcoholism, National Institutes of Health, Bethesda, MD, USA

2 Division of Psychiatry, University of Edinburgh, Royal Edinburgh Hospital, Edinburgh, UK

3 MRC Integrative Epidemiology Unit at the University of Bristol, Bristol, UK

\section{Introduction}

Alcohol consumption is a major risk factor exhibiting a complex relationship with death and disability in the United States and worldwide with the World Health Organization estimating alcohol is responsible for 139 million disabilityadjusted life-years globally [1-3]. Acute intoxication may result in injuries, poisoning, and interpersonal violence [3, 4], while longer-term alcohol consumption contributes to chronic diseases, including cancer [5-7], cardiovascular disease [8-10], and dependent drinking exacerbating psychiatric comorbidities or other impairments [2,11]. The complex relationship between alcohol and morbidity is due, in part, to the pattern of its use and the beverage type consumed $[12,13]$ with beer and hard liquor consumption associated with more severe drinking patterns and increased risk for alcohol-related 
problems [14]. In addition, while the quantity of alcohol consumed and alcohol intake frequency are correlated [15], and often used interchangeably, they demonstrate different and often opposite effects on health [15]. Using genetic correlations, Marees et al. [15] recently showed opposing associations of alcohol quantity and intake frequency with many health behaviors, including smoking, various psychiatric disorders, and personality traits, suggesting different risk profiles related to these alcohol consumption metrics.

The suggested differences in risk profiles with different alcohol consumption patterns and the seriousness of the acute and chronic diseases linked with risky alcohol consumption highlights the importance of identifying causal risk factors related to how alcohol is consumed to develop and improve intervention and treatment strategies. Among various social determinants associated with health disparities (age, gender, race, ethnicity, etc.) and mortality, educational attainment (EA) has been identified as a prominent risk factor [16]. For example, at age 25, the average life expectancy of U.S. adults without a high-school diploma is 9 years shorter than college graduates [17]. The impact of education on alcohol consumption behaviors may be an important pathway mediating these effects. Observational studies have demonstrated EA likely influences drinking patterns, beverage preferences, and alcohol-related outcomes [13, 18, 19]. Higher EA is associated with reduced odds of reporting high-risk drinking $[1,18]$, or at least one episode of heavy episodic drinking within the past twelve months [13, 17, 20]. Moreover, individuals with fewer years of education are more likely to report higher single-occasion quantity consumed and alcohol-related harm [17]. There is also conflicting evidence that alcohol consumption associates with EA with some studies showing an association with decreased years of schooling while others find either very small or non-significant effects [21]. While education is associated with differences in alcohol consumption behaviors, observational studies are subject to reverse causation, or residual confounding [22-24]. Recent genetic studies have suggested that the relationship between alcohol use and EA is complex [15] and differs markedly depending on which aspect of alcohol use is considered. Sanchez-Roige et al. [25] observed a positive genetic correlation $\left(r_{\mathrm{g}}\right)$ between college completion and Alcohol Use Disorder Identification Test (AUDIT) total scores $\left(r_{\mathrm{g}}=0.23\right.$; standard error $(\mathrm{se})=$ 0.05) [26]; Walters et al. [27] observed a negative genetic correlation between EA and DSM-IV alcohol dependence (AD) $\left(r_{\mathrm{g}}=-0.47\right.$; se $\left.=0.07\right)$; and Marees et al. [15] found a positive genetic correlation between EA and alcohol intake frequency [26, 28], but a negative correlation between EA and total alcohol intake quantity [27] (genetic correlations for the current study are presented in Supplementary Table 1). Furthermore, inferring causality from correlations and multivariable adjusted regression models is often unreliable $[29,30]$.
Mendelian randomization (MR) analysis uses randomly inherited genetic markers (single nucleotide polymorphisms (SNPs)) robustly associated with a risk factor (e.g., education) as proxies for environmental exposures to assess causal inferences about the effect of the exposure on an outcome (e.g., alcohol consumption patterns and AD risk). MR has some analogies to randomized controlled trials (RCTs) since genetic variants are not modifiable and free from reverse confounding [23, 24, 31] and is an important strategy for establishing evidence of causal relationships where RCTs are impractical or unethical [23]. The increasing availability of summary-level data from genomewide association studies (GWASs) can be used to perform MR analyses where gene exposure and gene outcome measures are derived from two separate GWAS [32]. These two-sample MRs benefit from increased statistical power and enable sensitivity analyses to test the robustness of the MR findings [33].

Recent two-sample MR studies have shown inverse relationships between EA and both smoking and coronary heart disease [34, 35]. However, to our knowledge, MR has not been applied to examine the effects of EA on alcohol consumption patterns, DSM-IV alcohol dependence, or other indicia of alcohol use disorders. In this study, using the largest, publicly available GWASs to date, we conducted a bidirectional two-sample MR of EA $(N=293,723)$ [36] on $\operatorname{AD}(N=28,657)$ [27], AUDIT scores $(N \leq 121,604)$ $[25,26]$, and alcohol consumption (total quantity consumed [37], intake frequency, whether alcohol is consumed with meals, and drink-specific average weekly intake (cider and beer, red and white wine, and distilled spirits)) $(N \leq$ 462,346) [38] to assess the evidence of causal associations between EA and alcohol dependence and consumption. Given men and women differ in their alcohol consumption patterns and alcohol-related problems [39], we also performed exploratory two-sample MR analyses using sexspecific alcohol consumption and AUDIT GWASs ( $N$ (females) $\leq 194,174 ; N$ (males) $\leq 167,010$ ), where available, to evaluate whether EA differentially impacts drinking behaviors between men and women.

\section{Methods}

\section{Data sources}

GWASs included in the current study are described in Table 1. We selected online publicly available GWASs with the largest sample sizes consisting of populations of European ancestry and without significant sample overlap. Details of the GWASs, including quality control and association methods, are available in Supplementary Methods 1 . All GWASs have existing ethical permissions from their 
Table 1 GWASs included in the current study

\begin{tabular}{|c|c|c|c|c|}
\hline Phenotype & Source & Citation & Sample size & Variable \\
\hline Educational attainment $(\mathrm{SD}=3.61$ years $)$ & SSGAC & Okbay et al. [36] & 293,723 & Continuous \\
\hline Average before tax household income & Neale Lab UKB & www.nealelab.is/uk-biobank & 411,028 & Categorical \\
\hline \multicolumn{5}{|l|}{ Alcohol use: } \\
\hline Alcohol intake frequency & MRC-IEU UKB & Elsworth et al. [38] & 462,346 & Categorical \\
\hline Weekly intake (drinks per week) & SSGAC & Karlsson Linnér [37] & 414,343 & Integer \\
\hline \multicolumn{5}{|l|}{ Weekly intake by drink type (units): } \\
\hline Distilled spirits (measure) & MRC-IEU UKB & Elsworth et al. [38] & 326,565 & Categorical \\
\hline Beer plus cider (pint) & MRC-IEU UKB & Elsworth et al. [38] & 327,634 & Categorical \\
\hline Red wine (glass) & MRC-IEU UKB & Elsworth et al. [38] & 327,026 & Categorical \\
\hline Champagne plus white wine (glass) & MRC-IEU UKB & Elsworth et al. [38] & 326,801 & Categorical \\
\hline \multicolumn{5}{|l|}{ Alcohol dependence (AD): } \\
\hline Alcohol dependence (DSM-IV diagnosis) & PGC & Walters et al. [27] & 28,657 & Binary \\
\hline Alcohol use disorders identification test (AUDIT): & & www.nealelab.is/uk-biobank & & \\
\hline Frequency of alcohol intake & Neale Lab UKB & & 117,914 & Categorical \\
\hline Amount of alcohol drunk on a typical drinking day & Neale Lab UKB & & 108,256 & Categorical \\
\hline Frequency of consuming $\geq 6$ or more units of alcohol & Neale Lab UKB & & 108,485 & Categorical \\
\hline Frequency of inability to cease drinking in the last year & Neale Lab UKB & & 67,973 & Categorical \\
\hline $\begin{array}{l}\text { Frequency of failure to fulfill normal expectations due to alcohol } \\
\text { (past year) }\end{array}$ & Neale Lab UKB & & 65,054 & Categorical \\
\hline Frequency of needing a morning drink & Neale Lab UKB & & 65,099 & Categorical \\
\hline $\begin{array}{l}\text { Frequency of feeling guilt or remorse after drinking alcohol } \\
\text { (past year) }\end{array}$ & Neale Lab UKB & & 65,009 & Categorical \\
\hline Frequency of memory loss due to drinking alcohol (past year) & Neale Lab UKB & & 65,029 & Categorical \\
\hline Ever been injured or injured someone else & Neale Lab UKB & & 118,002 & Categorical \\
\hline Ever had a known person concerned or recommend reduction & Neale Lab UKB & & 117,880 & Categorical \\
\hline
\end{tabular}

respective institutional review boards and include participant informed consent.

\section{Genetic instruments for exposure: educational attainment}

We extracted summary association statistics for the 74 genome-wide significant $\left(P<5 \times 10^{-8}\right)$ SNPs previously demonstrated to be associated with EA defined as years of schooling [36], measured in standard deviation (sd) units (mean $=14.33$ years, $s d=3.61$ years), ascertained in up to 293,723 persons from 64 discovery cohorts (excluding the subsequent UKB replication cohort). Twenty-one of the 74 SNPs were excluded by linkage disequilibrium (LD) of $R^{2}=0.001$ and clumping distance $=10,000 \mathrm{~kb}$, leaving 53 independent SNPs for the two-sample MR analysis (Supplementary Table 2).

EA is highly heritable and strongly genetically correlated with markers of socioeconomic status (SES) [40]. Non-zero genetic correlations suggest shared genetic factors contributing to these social outcomes [40]. We sought to account for the effects of SES, as operationalized by income, by performing additional analyses with an EA instrument constructed by removing variants associated with income. We took advantage of the recent average household before tax income GWAS $(N=$ 311,028 ) from the Neale Lab UK Biobank (UKB) GWAS (http://www.nealelab.is/uk-biobank/), generating an ordinal categorical phenotype (sample frequency in \%): $<18,000 £ \quad(21.8 \%), \quad 18,000-30,999 £ \quad(25.4 \%)$, $31,000-51,999 £(26.4 \%), 52,000-100,000 £$ (21.9\%), and $>100,000 £(5.5 \%)$. We removed variants from the EA instrument associated with income at a threshold significance of 0.00094 (nominal $P=0.05$ corrected for 53 comparisons, the number of SNPs in the main EA instrument), leaving 30 independent variants (Supplementary Table 3).

\section{Genetic instruments for outcomes}

\section{Alcohol consumption}

We used summary statistics from the MRC-IEU UKB GWAS Pipeline [38] on six alcohol consumption behaviors generated using the PHEnome Scan Analysis Tool (PHESANT) [41] as ordinal categorical responses: (1) alcohol 
intake frequency, i.e., never, special occasions only, one to three times a month, once or twice a week, three or four times a week, daily or almost daily $(N=432,346)$; and for the subset of UKB respondents who indicated they drank at least once or twice a week, also assessed were (2) average weekly spirits intake in increments of one pub measure of alcohol $(N=326,565)$; (3) average weekly beer plus cider intake in increments of one pint $(N=327,634)$; (4) average weekly red wine intake in increments of one glass $(N=$ 327,026); (5) average weekly white wine plus champagne in increments of one glass $(N=326,801)$; and (6) average weekly fortified wine intake in increments of one glass $(N=327,563)$. We also used summary association statistics from the MRC-IEU UKB GWAS Pipeline on an additional alcohol consumption behavior assessed as a binary response $(0=$ No, $1=$ Yes $)$ : (7) alcohol usually taken with meals $(N$ cases $=159,104 ; N$ controls $=75,541)$. For the additional sex-specific analyses, we used statistics from sexspecific GWASs from the Neale Lab UKB GWAS: sexspecific GWASs were not available from the MRC-IEU UKB GWAS Pipeline. Out of the 53 possible independent SNPs associated with EA, 52 were present in these MRCIEU GWASs, 1 SNP was identified in high linkage disequilibrium (LD) as a proxy for the missing SNP, and 2 SNPs were removed for being palindromic with intermediate allele frequencies (to harmonize the data so that the effect of the variants on the exposure EA and outcomes corresponded to the same allele), leaving 51 SNPs for analysis (Supplementary Table 2).

For a measure of total alcohol consumption, we used summary statistics from the Social Science Genetics Association Consortium (SSGAC) GWAS of alcohol consumption in the UKB $(N=414,343)$, measured as "drinks per week" (DPW), constructed, for UKB participants who indicated they drank "at least once or twice per week", by aggregating the weekly intake of distilled spirits (pub measures), beer and cider (pints), red wine, white wine, and champagne (glasses), and other alcoholic drinks, e.g., alcopops (DPW: mean $=8.92$ drinks, $\mathrm{SD}=9.30$ drinks) [37]. For UKB participants who indicated they drank "one to three times a month", the phenotype was constructed by aggregating the monthly intake over all drink types and dividing by four. Sex-specific alcohol consumption GWASs were not available from the SSGAC. Out of the 53 possible SNPs associated with EA, 52 were present in this SSGAC GWAS, and 2 SNPs were removed for being palindromic with intermediate allele frequencies, leaving 50 SNPs (Supplementary Table 2).

\section{Alcohol dependence and alcohol use disorders identifiers}

We used summary association statistics from the PGC GWAS on AD, defined as meeting criteria for a Diagnostic and Statistical Manual of Mental Disorders IV (DSM-IV, or DSM-IIIR in one instance) diagnosis, in 28,657 participants (cases $N=8,485$; controls $N=20,657$ ) [27]. Sex-specific AD GWASs were not available. Out of the 53 possible SNPs associated with EA, 53 were present in this PGC GWAS, and 9 SNPs were removed for being palindromic with intermediate allele frequencies, leaving 44 SNPs (Supplementary Table 2).

To assess identifiers or symptoms of alcohol dependence or use, we used summary association statistics from the Neale Lab UKB GWAS (http://www.nealelab.is/uk-bioba $\mathrm{nk} /$ ) for responses to the ten-item AUDIT; for the supplementary sex-specific analyses, we used statistics from the corresponding sex-specific GWASs. The PHESANT [41] generated phenotype categories are further described in Supplementary Table 4 and Supplementary Methods 2. Out of the 53 possible SNPs associated with EA, 53 were present in these ten Neale Lab GWASs, and 8 SNPs were removed for incompatible alleles, leaving 45 SNPs (Supplementary Table 2).

\section{Bidirectional analysis}

We extracted exposure summary association statistics for genome-wide significant $\left(P<5 \times 10^{-8}\right)$ SNPs associated with the alcohol consumption, AD and AUDIT GWASs described above, removed SNPs in LD with other SNPs, then extracted outcomes in the EA GWAS, harmonized exposure and outcomes, removing palindromic alleles with intermediate frequencies, for the bidirectional two-sample MR analyses. Further details are described in Supplementary Methods 3.

\section{Sample overlap}

Participant overlap between the samples used to estimate genetic associations between exposure and outcome in twosample MR can bias results [42]. We endeavored to use only non-overlapping GWAS summary statistics to reduce this source of bias. We used the discovery EA GWAS $(N=$ 293,723), including the $23 \&$ Me cohort but excluding the UKB replication cohort; thus, there was no overlap between the exposure cohorts and alcohol consumption and AUDIT outcomes (solely UKB cohorts). For alcohol dependence, a comparison of the cohorts included in the PGC alcohol dependence GWAS and SSGAC EA GWAS showed two common cohorts $(N=11,096)$ (Minnesota Center for Twin and Family Research; Swedish Twin Registry). The relevant percentage overlap for purposes of determining weak instrument bias (WIB) is taken with respect to the larger data set - only the presence of participants in both studies leads to correlation in estimates [42]. Here, the two common cohorts accounted for $3.8 \%$ of participants in the larger 
SSGAC GWAS; based on simulation studies of the association between sample overlap and the degree of WIB, considerable bias is not expected [42].

\section{Statistical analysis}

\section{Genetic correlation}

We estimated SNP heritability as well as cross-trait genetic correlation between EA and alcohol consumption and dependence by linkage disequilibrium score regression (LDSR) [43], using summary-level statistics from the previously conducted GWASs, all based on large samples. We used the centralized database and web interface, LD Hub [44]. Analysis was restricted to well-imputed SNPs for the selected phenotypes, with SNPs filtered to HapMap3 SNPs with 1000 Genomes EUR minor allele frequency (MAF) above $5 \%$, and insertions and deletions, structural variants, strand-ambiguous, and unmatched SNPs removed, along with SNPs within the major histocompatibility complex region, and SNPs with extremely large effect sizes [44]. Significant genetic correlations within the UKB cohort were identified by applying a Bonferroni correction for 20 crosstrait comparisons (threshold $P<0.0025$ ) (Supplementary Table 1).

\section{Two-sample Mendelian randomization}

We used four complementary methods-inverse-variance weighted (IVW) MR, MR Egger, weighted median, and weighted mode MR-to assess evidence of the association of EA and the risks of alcohol use behaviors and alcohol use disorders and also discern sensitivity to different patterns of violations of instrumental variable (IV) assumptions [33]. We reference IVW MR for the main results: in the absence of pleiotropy and assuming the instruments are valid, IVW MR estimates are the best unbiased estimates [45]. Consistency of results across these methods (each making different assumptions about pleiotropy) strengthens causal inference; significant divergent results may indicate bias from genetic pleiotropy.

To evaluate heterogeneity in instrument effects, which may indicate potential violations of the IV assumptions underlying two-sample MR [46], we used both MR Egger intercept test [46] and the Cochran heterogeneity test [47]. We also used the MR pleiotropy residual sum and outlier (MR-PRESSO) global test [48] to identify outlier variants for removal to correct potential directional horizontal pleiotropy and resolve detected heterogeneity. We include an overview of the analyses in Fig. 1. Details about these MR methods and tests are included in Supplementary Methods 5. We used the Steiger directionality test to test the causal direction between the hypothesized exposure and outcomes [49]. Given a nominal threshold of 0.05 , and 20 comparisons in the UKB sample, we apply a Bonferroni corrected threshold of $P=0.0025$. Analyses were carried out using TwoSampleMR, version 4.16 [33], and MRPRESSO, version 1.0 [50], in the R environment, version 3.5.1 (02-07-2018).

\section{Results}

\section{Overview}

We present the genetic correlation results from LDSR in Supplementary Table 1. As regards two-sample MR, we report those estimates (1) agreeing in direction and magnitude across MR methods, exceeding nominal significance $(P<0.01)$ in IVW MR, $(2)$ not indicating bias from horizontal pleiotropy (MR-PRESSO global test $P>0.01$ ), nor directional pleiotropy (MR Egger intercept $P>0.01$ ), and (3) indicating true causal effect directionality (Steiger directionality test $P<0.01$ ), except where otherwise noted. We present the outlier corrected MR estimates in Figs. 2 and 3; individual genetic variant associations in Supplementary Table 2; MR results in Supplementary Tables 5-7; bidirectional MR results in Supplementary Table 8; and in Supplementary Table 9, single-SNP and leave-one-out results for the main analyses.

\section{Genetic correlations from LDSR}

EA showed significant genetic correlations (Bonferroni corrected $P<0.0025$ for 20 comparisons within the UKB cohort) with all of the alcohol intake (quantity) traits: positive weak correlation with total drinks per week $\left(r_{\mathrm{g}}=\right.$ $0.092, P=4.75 \times 10^{-5}$ ), but negative weak correlation with amount of alcohol drunk on a typical drinking day (AUDIT question 2: $\left.r_{\mathrm{g}}=-0.240, P=1.89 \times 10^{-10}\right)$; as well as correlations, in different directions, of EA with alcohol intake by drink type: positive strong correlations with average weekly intake of champagne plus white wine $\left(r_{\mathrm{g}}=\right.$ $\left.0.654, P=5.86 \times 10^{-109}\right)$ and red wine $\left(r_{\mathrm{g}}=0.609, P=\right.$ $\left.9.99 \times 10^{-154}\right)$, negative moderate correlations with average weekly intake of distilled spirits $\left(r_{\mathrm{g}}=-0.372, P=8.06 \times\right.$ $\left.10^{-32}\right)$ and beer plus cider $\left(r_{\mathrm{g}}=-0.409, P=2.79 \times 10^{-40}\right)$. We also observed significant positive genetic correlations of EA with a subset of alcohol intake frequency traits: moderate correlation with alcohol intake frequency $\left(r_{\mathrm{g}}=0.465\right.$, $\left.P=1.99 \times 10^{-133}\right)$, strong correlation with alcohol usually taken with meals $\left(r_{\mathrm{g}}=0.622, P=1.67 \times 10^{-186}\right)$, but none with frequency of consuming $\geq 6$ alcohol units.

EA also showed significant negative moderate genetic correlation with the $\mathrm{AD}$ risk $\left(r_{\mathrm{g}}=-0.463, P=6.82 \times 10^{-10}\right)$; but weak correlations, in opposite directions, with two of four 


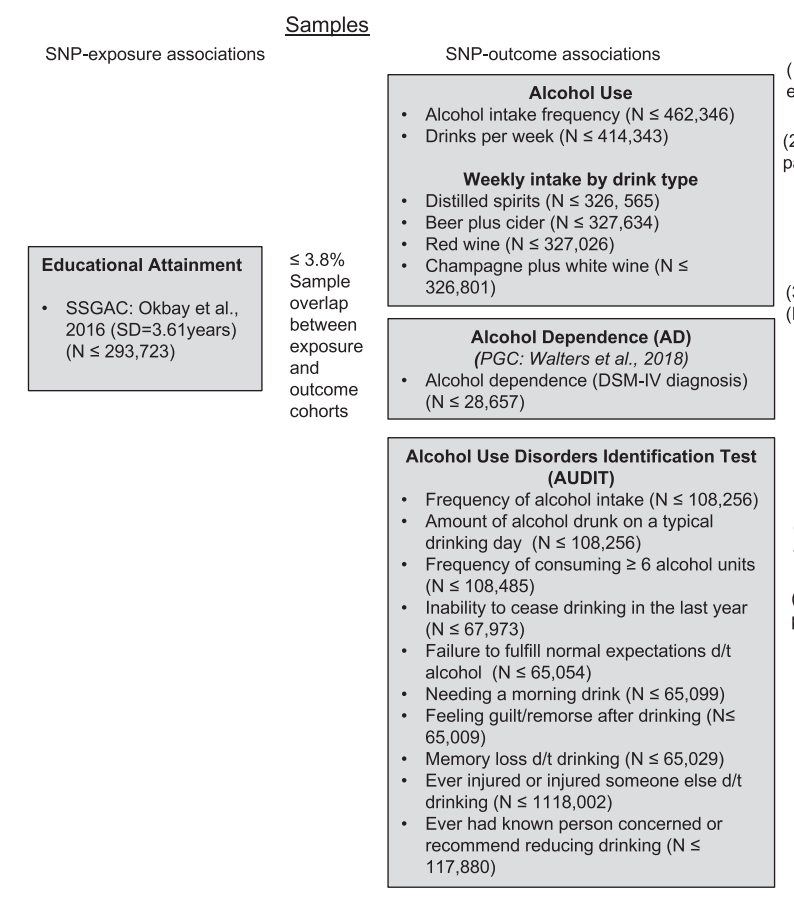

Fig. 1 Overview of the main analysis. SSGAC $=$ Social Science Genetics Association Consortium; PGC $=$ Psychiatric Genomics Consortium; MRC-IEU = Medical Research Council Integrative Epidemiological Unit, University of Bristol; GWAS = genome-wide association study; $\quad \mathrm{UKB}=\mathrm{UK}$ Biobank; $\mathrm{AIF}=$ alcohol intake

AUDIT responses related to problematic or hazardous alcohol use (frequency of feeling guilt or remorse after drinking (past year): $r_{\mathrm{g}}=0.164, P=0.0007$; frequency of memory loss due to drinking alcohol (past year): $r_{\mathrm{g}}=-0.203, P=0.0003$ ). We did not observe evidence for a significant genetic correlation of EA and the three AUDIT responses related to AD. We did observe a strong positive correlation of EA with average household income before tax $\left(r_{\mathrm{g}}=0.805, P<0.001\right)$. See Supplementary Table 1.

\section{Effects of educational attainment on alcohol consumption and consumption frequency}

\section{Weekly alcohol intake}

Genetic variants associated with increased EA were not significantly associated with total number of drinks per week (sum total of different types of drinks) $\left(\beta_{\mathrm{IVw}}=0.031\right.$, $95 \% \mathrm{CI},-0.015$ to $0.076, P_{\mathrm{IVW}}=0.189$ ). In the subsample of UKB participating in the AUDIT module, variants associated with increased EA, however, were associated with decreased alcohol intake on a typical drinking day (AUDIT question 2: $\beta_{\mathrm{IVW}}=-0.207,95 \% \mathrm{CI},-0.293$ to $-0.120, \quad P_{\mathrm{IVW}}=2.87 \times 10^{-6}$ ), disaggregating DPW by drink type, variants associated with increased EA were also associated with decreased average weekly spirits intake $\left(\beta_{\mathrm{IVW}}=-0.148,95 \% \mathrm{CI},-0.188\right.$ to $-0.107, P_{\mathrm{IVW}}=$
Instrument Selection \& Outlier Correction

Methods

Heterogeneity

(1) Instrument selection: 53 independent variants associated ducational attainment $(\mathrm{EA})$ at $\mathrm{P}<5 \times 10^{-8}\left(\mathrm{LD} \mathrm{r}^{2}=.001,10,000 \mathrm{kB}\right)$ 2) Harmonize with available SNPs in outcome datasets, and remove palindromic SNPs (no. SNPs removed)

AlF $\&$ DPW
$(2$ removed $)$$\quad \begin{gathered}\text { AD } \\ (9 \text { removed })\end{gathered} \begin{gathered}\text { AUDIT } \\ \text { questions } \\ \text { (8 removed })\end{gathered} \begin{gathered}\text { DPW by drink } \\ \text { type } \\ (2 \text { removed })\end{gathered}$

(3) Apply MR-PRESSO global heterogeneity test and remove outliers nal no. SNPs)

\begin{tabular}{|c|c|c|}
\hline $\begin{array}{c}\text { AIF \& DPW } \\
\text { (42 \& 47 } \\
\text { SNPs) }\end{array}$ & $\begin{array}{c}\mathrm{AD} \\
(44 \mathrm{SNPs})\end{array}$ & $\left(\begin{array}{c}\text { AUDIT } \\
\text { questions } \\
(41-45 \text { SNPs) }\end{array}\right.$ \\
\hline
\end{tabular}

\section{VW}

MR Egger Intercept

Weighted median Cochran Q Weighted mode MR-PRESSO

Instrument Selection Accounting for Household ncome (Neale Lab UKB GWAS, $\mathrm{N} \leq 311.028$ )

4) Remove 23 of 53 independent EA variants nominally associated SNPs ( $P$ corrected for 53 comparisons: $0.05 / 53=0.0009$ )

(5) Harmonize with available SNPs in outcome datasets, and remove alindromic SNPs (no. SNPs removed)

$\left.\begin{array}{c}\begin{array}{c}\text { AIF } \& \text { DPW } \\ (1-2 \\ \text { removed })\end{array} \\ \text { AD } \\ \text { (none) }\end{array}\right] \begin{gathered}\text { AUDIT } \\ \text { questions } \\ (4 \text { removed })\end{gathered} \quad \begin{gathered}\text { DPW by drink } \\ \text { type } \\ (0-2 \text { removed })\end{gathered}$

(6) Apply MR-PRESSO global heterogeneity test and remove outliers nal no. SNPs)

AIF \& DPW $(20 \mathrm{SNPs})$

\begin{tabular}{|c|c|c|}
\hline $\begin{array}{c}\mathrm{AD} \\
(20 \mathrm{SNPs})\end{array}$ & $\begin{array}{c}\text { AUDIT } \\
\text { questions } \\
\text { (20-24 SNPs) }\end{array}$ & $\begin{array}{c}\text { DPW by drink } \\
\text { type } \\
\text { (24-30 SNPs) }\end{array}$ \\
\hline
\end{tabular}

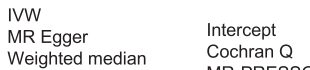

Cochran Q

frequency; $\mathrm{DPW}=$ drinks per week; $\mathrm{AD}=$ alcohol dependence; AUDIT $=$ Alcohol Use Disorder Inventory Test; $\mathrm{SNP}=$ singlenucleotide polymorphism; $N=$ sample size; $\mathrm{MR}=$ Mendelian randomization; IVW = inverse-variance weighted Mendelian randomization

$\left.6.24 \times 10^{-13}\right)$ and weekly beer plus cider intake $\left(\beta_{\mathrm{IVW}}=\right.$ $-0.178,95 \% \mathrm{CI},-0.217$ to $-0.140, P_{\mathrm{IVW}}=5.58 \times 10^{-20}$ ), although outlier correction notwithstanding, evidence of residual heterogeneity and horizontal pleiotropy for beer plus cider intake did remain. Conversely, variants associated with increased EA were associated with increased average weekly champagne plus white wine intake $\left(\beta_{\mathrm{IVW}}=\right.$ $0.199,95 \%$ CI, $\left.0.159-0.238, P_{\mathrm{IVw}}=7.96 \times 10^{-23}\right)$, as well as increased average weekly fortified wine $\left(\beta_{\mathrm{IVW}}=0.050\right.$, 95\% CI, 0.027-0.073, $P_{\mathrm{IVW}}=1.87 \times 10^{-5}$ ); and increased average weekly red wine intake $\left(\beta_{\mathrm{IVW}}=0.204,95 \% \mathrm{CI}\right.$, $\left.0.161-0.248, \quad P_{\mathrm{IVW}}=6.67 \times 10^{-20}\right)$. See Supplementary Table 5.

\section{Alcohol intake frequency}

Genetic variants associated with increased EA were associated with increased alcohol intake frequency $\left(\beta_{\mathrm{IVW}}=0.331\right.$, 95\% CI, 0.267-0.396, $P_{\mathrm{IVW}}=4.62 \times 10^{-24}$; see also AUDIT question 1: $\beta_{\mathrm{IVW}}=0.197,95 \% \mathrm{CI}, 0.083-0.311$, $P_{\mathrm{IVW}}=7.30 \times 10^{-4}$ ). Variants associated with increased EA were also associated with decreased frequency of consuming six or more units of alcohol per occasion (AUDIT question 3: $\beta_{\mathrm{IVW}}=-0.198,95 \% \mathrm{CI},-0.297$ to 0.099 , $P_{\mathrm{IVW}}=9.14 \times 10^{-5}$ ), and also with an increased probability of drinking alcohol with meals $\left(\beta_{\mathrm{IVW}}=0.174,95 \% \mathrm{CI}\right.$, $\left.0.141-0.208, P_{\mathrm{IVW}}=1.63 \times 10^{-24}\right)$, although evidence of 


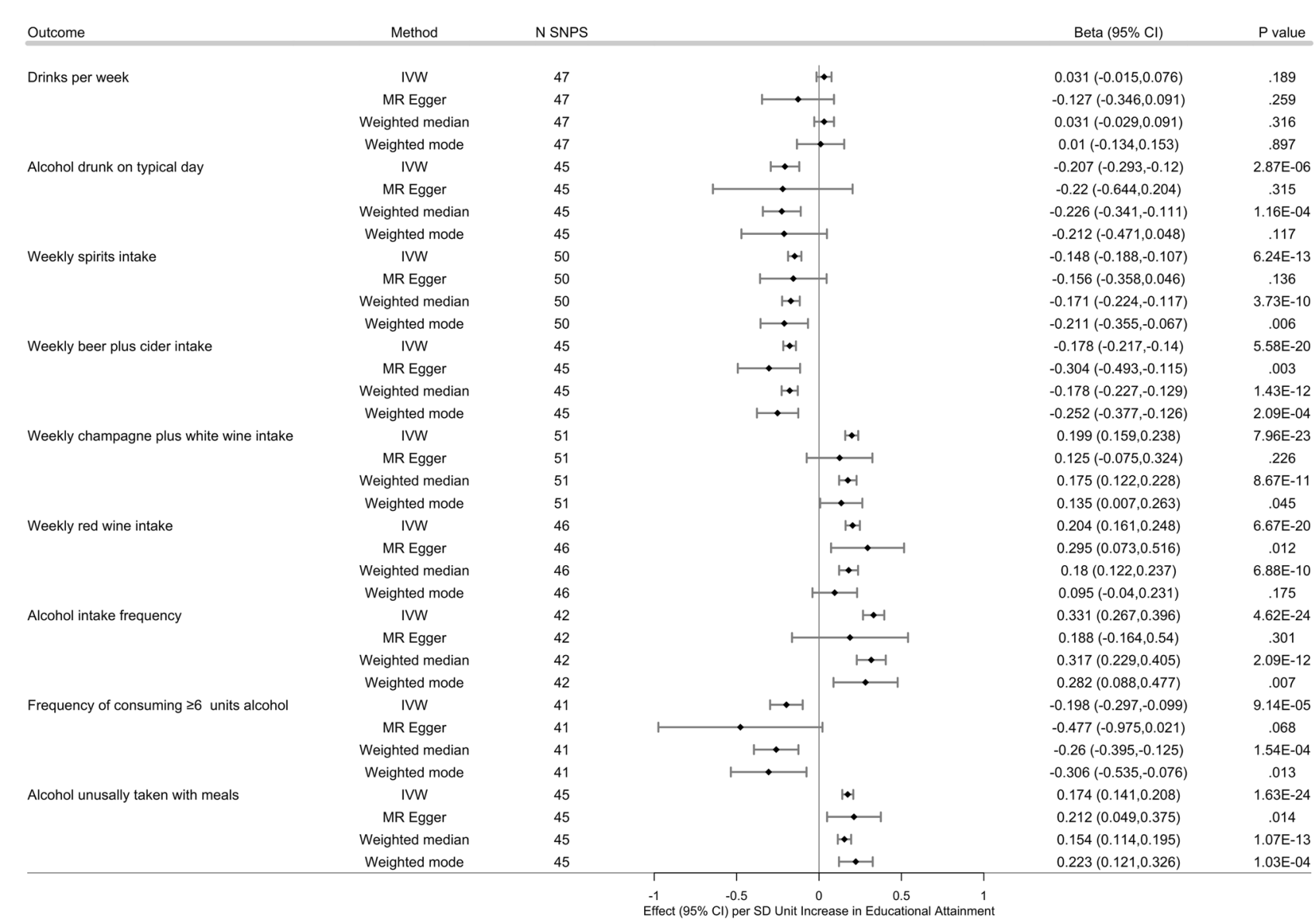

Fig. 2 Effects of the genetic variants for increased educational attainment (EA) on alcohol use. Fifty-three genome-wide significantly associated $\left(P<5 \times 10^{-8}\right)$ independent (LD $R^{2}=0.001$, clumping distance $=10,000 \mathrm{~kb}$ ) single nucleotide polymorphisms (SNPs) were used as instruments for EA. Results from inverse-variance weighted (IVW) and three complementary two-sample MR methods, following removal of variants identified as outliers (MR-PRESSO $P<0.10$ ), are

residual heterogeneity and horizontal pleiotropy did remain (Supplementary Table 5).

\section{Alcohol intake and intake frequency accounting for income}

Removing EA instruments associated with average household income attenuated the significance, and reduced heterogeneity, but with only one exception, did not significantly affect the magnitude nor direction of the associations. Variants associated with increased EA, but not associated with household income, were associated with increased average weekly red wine intake, but with a smaller effect size $\left(\beta_{\mathrm{IVW}}=0.123,95 \% \mathrm{CI}, 0.064-0.181\right.$, $\left.P_{\mathrm{IVW}}=3.80 \times 10^{-5}\right)($ Supplementary Table $6 \mathrm{~A})$.

\section{Sex-specific intake and intake frequency}

Exploratory sex-specific analyses were motivated by these results, i.e., EA differentially associated with average weekly shown. Effect $(\beta)$ measures the change per unit increase in outcome per standard deviation ( $\mathrm{SD}=3.61$ years) increase in EA. Error bars indicate $95 \%$ confidence intervals at the nominal threshold 0.05 . With 20 comparisons overall in the UKB cohort, the Bonferroni corrected threshold for comparisons would be 0.0025 , given a nominal threshold $0.05 . \mathrm{LD}=$ linkage disequilibrium; $\mathrm{MR}=$ Mendelian randomization; $\mathrm{IVW}=$ inverse-variance-weighted $\mathrm{MR} ; \beta=$ effect estimate

intake by drink types, along with surveys finding drink of choice differs by sex, females preferring wine, then beer and spirits, and males preferring beer, then spirits, lastly wine [51]. Genetic variants associated with increased EA were differentially associated across sexes (non-overlapping CIs) with decreased average weekly spirits intake, with greater effect for females (female: $\beta_{\mathrm{IVW}}=-0.218,95 \% \mathrm{CI},-0.286$ to -0.150 , $P_{\mathrm{IVW}}=2.95 \times 10^{-10}$; male: $\beta_{\mathrm{IVW}}=-0.084,95 \% \mathrm{CI},-0.146$ to $-0.022, P_{\mathrm{IVW}}=7.65 \times 10^{-3}$ ); and also decreased average weekly beer plus cider intake, with, in contrast, greater effect for males (female: $\beta_{\mathrm{IVW}}=-0.115,95 \% \mathrm{CI},-0.165$ to $-0.065, P_{\mathrm{IVW}}=5.91 \times 10^{-6}$; male: $\beta_{\mathrm{IVW}}=-0.246,95 \% \mathrm{CI}$, -0.321 to $\left.-0.170, P_{\mathrm{IVW}}=1.75 \times 10^{-10}\right)$; but with increased average weekly intake of red wine, again with greater effect for males (female: $\beta_{\mathrm{IVW}}=0.161,95 \% \mathrm{CI}, 0.094-0.228$, $P_{\mathrm{IVW}}=2.61 \times 10^{-6} ; \quad$ male: $\quad \beta_{\mathrm{IVW}}=0.262, \quad 95 \% \quad \mathrm{CI}$, $\left.0.200-0.324, P_{\text {IVW }}=1.45 \times 10^{-16}\right)$. Variants associated with increased EA were associated with average weekly intake of white wine plus champagne for both females and males, with 


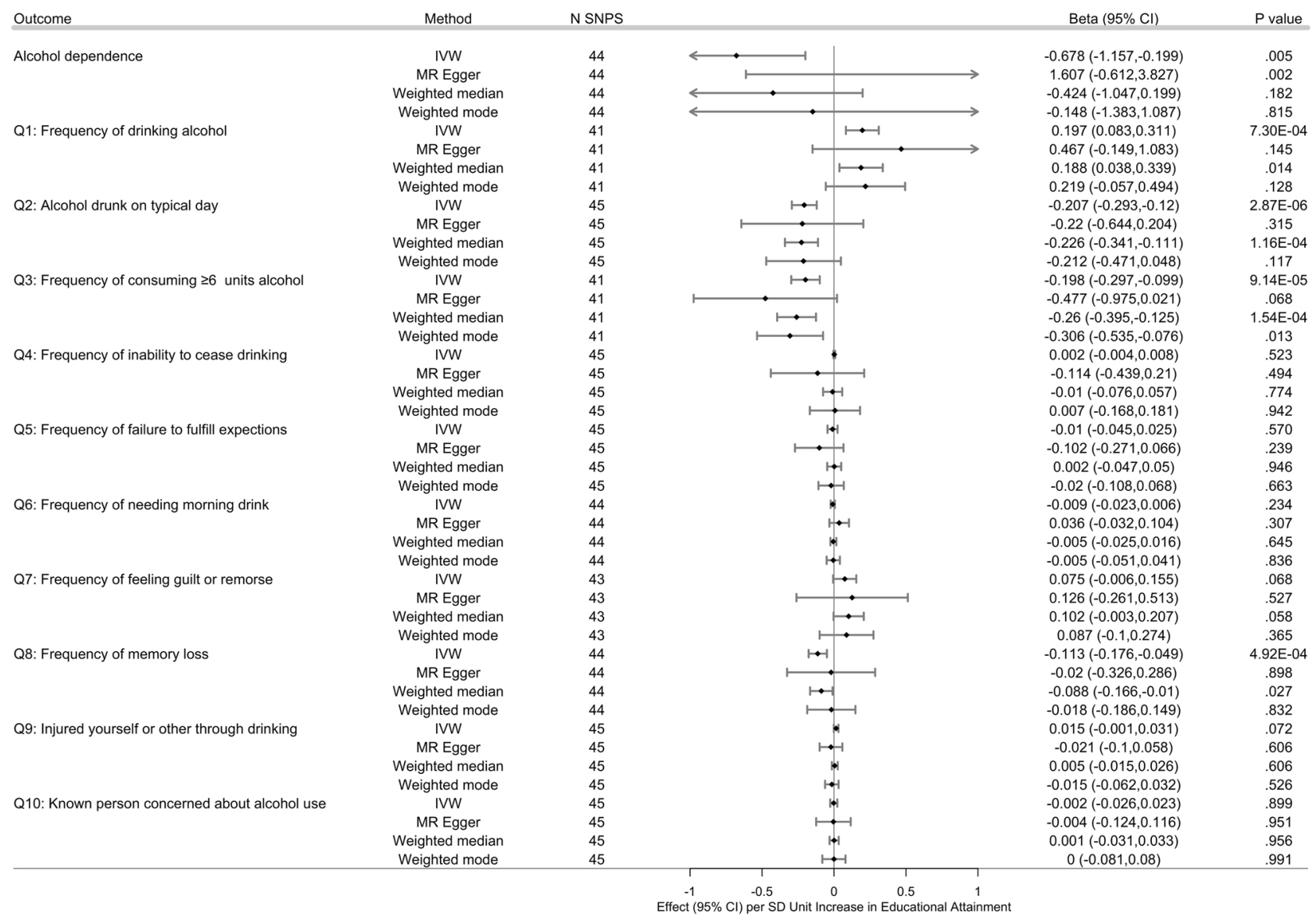

Fig. 3 Effects of the genetic variants for increased educational attainment (EA) on alcohol dependence (AD) and AUDIT. Fifty-three genome-wide significantly associated $\left(P<5 \times 10^{-8}\right)$ independent (linkage disequilibrium (LD) $R^{2}=0.001$, clumping distance $=$ $10,000 \mathrm{~kb}$ ) single nucleotide polymorphisms (SNPs) were used as instruments for EA. AUDIT outcomes were assessed on sub-cohort of UKB participants in the UKB AUDIT module. Results from inverse variance weighted (IVW) and three complementary twosample MR methods, following removal of variants identified as outliers (MR-PRESSO $P<0.10)$ are shown. Effect $(\beta)$ measures the

no significant difference in effect between sexes (overlapping CIs) (female: $\beta_{\mathrm{IVW}}=0.195,95 \% \mathrm{CI}, 0.129-0.261, P_{\mathrm{IVW}}=$ $5.97 \times 10^{-9}$; male: $\beta_{\mathrm{IVW}}=0.205,95 \% \mathrm{CI}, 0.144-0.266$, $\left.P_{\mathrm{IVW}}=5.16 \times 10^{-11}\right)$. See Supplementary Table 6B. Sexspecific GWASs for drinks per week were not available; however, in the subsample of UKB participants participating in the AUDIT module, variants associated with increased EA were associated with decreased alcohol intake on a typical drinking day for both females and males, again, with no significant difference between males and females (overlapping CIs) (AUDIT question 2: female $\beta_{\mathrm{IVW}}=-0.173$, $95 \%$ CI, -0.269 to $-0.077, P_{\mathrm{IVW}}=4.02 \times 10^{-4}$; male: $\beta_{\mathrm{IVW}}=-0.251,95 \% \mathrm{CI},-0.387$ to $-0.116, P_{\mathrm{IVW}}=2.86 \times$ $10^{-4}$ ). See Supplementary Table 6B.

Genetic variants associated with increased EA were associated with increased alcohol intake frequency for both change per unit increase in outcome per standard deviation $(\mathrm{SD}=3.61$ years) increase in $\mathrm{EA}$; with regard to binary outcome $\mathrm{AD}, \beta$ is equal to the ln (odds ratio) (OR) of AD per SD unit increase in EA. Error bars indicate $95 \%$ confidence intervals at the nominal threshold 0.05 . With 20 comparisons overall in the UKB cohort, the Bonferroni corrected threshold for comparisons would be 0.0025 , given a nominal threshold 0.05. With only one comparison in the PGC AD study, the threshold for $\mathrm{AD}$ is 0.05 . $\mathrm{EA}=$ Educational attainment; AUDIT $=$ Alcohol Use Disorder Identification Test: MR $=$ Mendelian randomization; IVW = inverse-variance weighted

females and males, but with no significant difference between sexes, and with evidence of residual heterogeneity and horizontal pleitropy (female: $\beta_{\mathrm{IVW}}=0.482,95 \% \mathrm{CI}$, $0.360-0.605, \quad P_{\mathrm{IVW}}=1.21 \times 10^{-14} ; \quad$ male: $\quad \beta_{\mathrm{IVW}}=0.317$, 95\% CI, $0.216-0.418, P_{\mathrm{IVW}}=6.81 \times 10^{-10}$ ). In contrast, variants associated with increased EA were associated with the probability of drinking alcohol with meals, and the effect was greater in males than females (female: $\beta_{\mathrm{IVW}}=$ $0.132,95 \%$ CI, $0.091-0.173, P_{\mathrm{IVW}}=2.50 \times 10^{-10}$; male: $\beta_{\mathrm{IVW}}=0.246,95 \% \mathrm{CI}, 0.186-0.305, P_{\mathrm{IVW}}=5.50 \times 10^{-16}$ ). See Supplementary Table 6B. For the subsample of UKB participants participating in the AUDIT module, variants associated with increased EA were associated with increased alcohol intake frequency for both females and males, with no significant difference between sexes (AUDIT question 1: female: $\beta_{\mathrm{IVW}}, 0.225,95 \% \mathrm{CI}$, 
0.063-0.388, $\quad P_{\mathrm{IVW}}=6.64 \times 10^{-3} ; \quad$ male: $\quad \beta_{\mathrm{IVW}}=0.143$, $95 \%$ CI, $\left.0.001-0.285, P_{\mathrm{IVW}}=0.048\right)$, and decreased frequency of consuming six or more units of alcohol per occasion, with attenuated significance for females, and a non-significant effect for males (AUDIT question 3: female: $\beta_{\mathrm{IVW}}=-0.183,95 \% \mathrm{CI},-0.303$ to -0.064 , $P_{\mathrm{IVW}}=.003$; male: $\beta_{\mathrm{IVW}}=-0.131,95 \% \mathrm{CI},-0.285$ to $\left.0.024, P_{\mathrm{IVW}}=.098\right)$. See Supplementary Table 6B.

\section{Effects of educational attainment on AD}

Genetic variants associated with increased EA were associated with decreased risk of $\mathrm{AD}$ by almost $50 \%$ per $\mathrm{SD}$ unit $\left(3.61\right.$ years schooling) (odds ratio $(\mathrm{OR})_{\mathrm{IVW}}=$ $0.508,95 \%$ CI, $\left.0.315-0.819, P_{\mathrm{IVW}}=5.52 \times 10^{-3}\right)$. Magnitude and direction of the causal estimates were consistent across IVW, weighted median, and weighted mode MR; the MR Egger point estimate, however, was directionally opposite but non-significant. See Supplementary Table 5. None of three AUDIT questions considered symptoms of $\mathrm{AD}$, i.e., frequency of inability to cease drinking, frequency of failure to fulfill normal expectations, and frequency of needing a morning drink, and only one of four AUDIT questions pertaining to problematic or hazardous alcohol use, i.e., decreased frequency of memory loss due to alcohol, were significantly associated with increased EA (question 8: $\beta_{\mathrm{IVW}}=-0.113,95 \% \mathrm{CI}$, -0.176 to $\left.-0.049, P_{\mathrm{IVW}}=4.92 \times 10^{-4}\right)$.

\section{$A D$ and AUDIT accounting for income}

Removing variants of EA associated with average household income attenuated the significance and reduced heterogeneity, but did not significantly affect the magnitude nor the direction of the associations (Supplementary Table 7A). Variants associated with increased EA, but not associated with household income, were associated with decreased risk of $\mathrm{AD}$ by still almost $50 \%$ (per unit $\mathrm{SD}=$ 3.61 years schooling $) \quad\left(\mathrm{OR}_{\mathrm{IVW}}=0.486, \quad 95 \% \quad \mathrm{CI}\right.$, $\left.0.241-0.980, \quad P_{\mathrm{IVW}}=0.044\right)$, and no longer with decreased frequency of memory loss due to alcohol (question $8, \beta_{\mathrm{IVW}}=-0.091,95 \% \mathrm{CI},-0.191$ to 0.009 , $\left.P_{\mathrm{IVW}}=0.755\right)$.

\section{Sex-specific AUDIT}

Genetic variants associated with increased EA were associated with decreased frequency of memory loss due to alcohol drinking in both females and males, but with no significant difference between sexes (question 8 , female $\beta_{\mathrm{IVW}}=-0.107,95 \% \mathrm{CI},-0.187$ to $-0.027, P_{\mathrm{IVW}}=0.008$; male: $\beta_{\mathrm{IVW}}=-0.127,95 \% \mathrm{CI},-0.199$ to $-0.055, P_{\mathrm{IVW}}=$ $\left.5.24 \times 10^{-4}\right)$ (Supplementary Table 7B).

\section{Bidirectional}

\section{Alcohol intake}

In supplementary bidirectional analyses, genetic variants associated with DPW were not associated with EA, but variants associated with increased average weekly intake of white wine plus champagne and red wine were associated with increased EA (white wine: $\beta_{\mathrm{IVW}}=1.021,95 \% \mathrm{CI}$, $0.765-1.278, P_{\mathrm{IVW}}=6.66 \times 10^{-15}$; red wine: $\beta_{\mathrm{IVW}}=0.753$, $95 \%$ CI, $0.627-0.880, P_{\mathrm{IVW}}=1.25 \times 10^{-31}$ ). Conversely, variants associated with beer plus cider intake but not spirits were associated with decreased EA (beer: $\beta_{\mathrm{IVW}}=-0.318$, $95 \% \mathrm{CI},-0.473$ to $-0.163, P_{\mathrm{IVW}}=5.79 \times 10^{-5}$; spirits: $\beta_{\mathrm{IVW}}=-0.154,95 \% \mathrm{CI},-0.408$ to $-0.098, P_{\mathrm{IVW}}=0.231$ ). Genetic variants associated with both increased alcohol intake frequency and increased frequency of drinking with meals were associated with increased EA (intake frequency: $\beta_{\mathrm{IVW}}=0.212,95 \% \mathrm{CI}, 0.174-0.251, P_{\mathrm{IVW}}=4.49 \times 10^{-27}$; meals: $\beta_{\mathrm{IVW}}=0.953,95 \% \mathrm{CI}, 0.786-1.119, P_{\mathrm{IVW}}=4.28 \times$ $10^{-29}$ ), but residual heterogeneity and horizontal pleiotropy remained after outlier correction. Genetic variants associated with increased AD and AUDIT were not significantly associated with EA. See Supplementary Table 8.

\section{Discussion}

Using large summary-level GWAS data and complementary two-sample MR methods, we show that EA has a likely causal relationship with alcohol consumption behaviors and alcohol dependence risk in individuals of European Ancestry. More specifically, higher EA reduced binge drinking (six or more units of alcohol), the amount of alcohol consumed per occasion, frequency of memory loss due to drinking, distilled spirits intake, and AD risk. EA increased the frequency of alcohol intake, whether alcohol is consumed with meals, and wine consumption. We found evidence that our results may be driven by genetic pleiotropy in only two of the eight alcohol consumption behaviors (average weekly beer plus cider intake and alcohol usually taken with meals) and significance remained after additional analysis using EA instruments with SNPs nominally associated with either cognition or income suggest that EA may be an important factor responsible for variation in alcohol use behaviors. Consistency of our results across MR methods also strengthens our inference of causality.

Educated persons generally have healthier lifestyle habits, fewer comorbidities, and live longer than their less educated counterparts [52], and our results suggest EA is causally associated with different likelihoods of belonging to variegated alcohol consumer typologies. We found that an additional 3.61 years of education reduced the risk of 
alcohol dependence by $\sim 50 \%$, which is consistent with results from small community samples [53], and the two most recent alcohol dependence GWASs findings strong inverse genetic correlations with educational attainment $[27,54]$. Notably, binge drinking significantly increases the alcohol dependence risk [55], and distilled spirits and beer consumption account for the majority of hazardous alcohol use [56]. Furthermore, compared to wine drinkers, beer and spirits drinkers are at increased risk of becoming heavy or excessive drinkers [57], for alcohol-related problems and illicit drug use [58, 59], and AD [57]. Our findings related to alcoholic drink preferences, when combined with our results showing increased binge drinking, memory loss due to alcohol, and a suggestive relationship with remorse after drinking, imply a pattern of alcohol consumption motivated to reduce negative emotions or becoming intoxicated [14].

In contrast to the often-reported positive association between EA and total amount of alcohol consumption reported from observational studies [18, 60], we found little evidence of a causal relationship. This null finding may be reconciled by the opposing influences on alcohol intake frequency and total alcohol consumed per occasion, which, while not leading to an overall change in total consumption, nonetheless significantly affect the pattern. Our null finding regarding total consumption does support similar results from Davies et al. [52], who used the 1972 mandated increase in school-leaving age in the UK as a natural experiment instrumental variable design to investigate the causal effects of staying in school on total alcohol consumption (from individuals in the UKB sample who turned 15 in the first year before and after the schooling age increased). Davies et al. may have found a significant effect of staying in school had they included the disaggregated behavioral dimensions of alcohol consumption behaviors. Nevertheless, even if no EA-total alcohol consumption relationship exists, studies have reported that both the specific alcoholic beverage and the pattern with which it is consumed, controlling for total consumption, independently contribute to risky health behaviors [61, 62].

Natural experiments [52, 63], and twin studies have found that differences in EA, even after controlling for shared environmental factors, still significantly impact mortality risk [64-66], and recent large Mendelian randomization studies have demonstrated inverse relationships between EA on smoking behaviors [35] and coronary heart disease (CHD) risk [34] add to the growing body of literature, suggesting a causal effect of increased EA on health and mortality. Other observational studies have linked alcohol consumption patterns to health, disease, and mortality risk [67-69]. In particular, binge drinking may have dramatic short-term consequences, including motor vehicle accidents, alcoholic coma, cerebral dysfunction, and violent behavior [70], as well as long-term effects such as hypertension, stroke, and other cardiovascular outcomes [71]. A recent MR study showed that smoking mediates, in part, the effect of education on cardiovascular disease [72], and our results suggest that differences in alcohol consumption patterns may also be another mediator. Health consequences incur significant costs with binge drinking accounting for $\sim 77 \%$ of the $\$ 249$ billion alcohol-related costs (lost workplace productivity, health care expenses, law enforcement, and criminal justice expenses, etc.) in the United States in 2010 [55].

While we do not fully understand the underlying biological mechanisms through which the instrument SNPs influence EA, they are primarily found in genomic regions regulating brain development and expressed in neural tissue. These SNPs demonstrate significant expression throughout the life course, but exhibit the highest expression during development [36]. For example, rs4500960, which was associated with reduced EA, is an intronic variant in the transcription factor protein, T-box, Brain 1 $(T B R 1)$, that is important for differentiation and migration of neurons during development [36], while rs10061788 is associated with cerebral cortex and hippocampal mossy fiber morphology [36]. It is, however, important to note that interpreting these SNPs as representing "genes for education" may be "overly simplistic" since EA is strongly affected by environmental factors [36]. Our results remained when using an EA instrument with SNPs nominally associated with income removed, suggesting that an individual's genetics may impact behavior development, which then increases EA [73]. Conversely, genetic estimates of EA and its correlations with other complex social phenotypes using population-based samples may be susceptible to biases, such as assortative mating and dynastic effects that provide pathways alternate to direct biological effects [40]. For example, EA-associated genetic influence on parental behavior could causally affect the child's environment [73]. Using polygenic scores for EA, Belsky et al. [73] recently found the mothers' EA-linked genetics actually predicted their children's social attainment better than the child's own EA-linked genetics, suggesting an effect mediated by environmental effects. While policies are not able to change children's genes, or their inherited social status, they can provide resources [73], and our results suggest that interventions to increase education may help improve health outcomes through changing alcohol consumption patterns.

Notably, there was evidence for some causal effects of alcohol consumption patterns on EA, and the divergent effects again demonstrate the importance of separating drinking variables. However, we failed to find evidence that total alcohol consumed, binge drinking, or AD impacts EA, which is in line with observational studies finding no, or small effects [21], and suggests that other studies findings a 
negative effect [21] may be due to confounding. Alternatively, EA may not be sensitive enough to detect changes in schooling, e.g., grade point average [21], falling behind in homework and other academic difficulties that also reported association with heavy drinking [74]. Further, there are currently no adolescent drinking behavior GWAS, so the temporal sequence of these analyses should be considered during their interpretation. Our findings, therefore, need replication when GWAS on adolescent alcohol consumption patterns becomes available.

Exploratory sex-specific analyses revealed differences in certain aspects of the relationship between EA and alcohol consumption. For men, the relationship between their consumption of red wine, beer, and whether they drink with meals was more sensitive to changes in EA than for women. Conversely, the reduction in binge drinking with increased EA may be driven by its effect for women since its effect on men was not significant. In addition, in women the negative effect of EA on spirit consumption was more than double its effect on men. We found no differences among the AUDIT question.

There are noted gender gaps in alcohol use and associated outcomes due to a combination of physiological and social factors [39]. Notably, Huerta et al. [75] found sexspecific effects of EA and academic performance on the odds of belonging to different alcohol consumption typologies (ranging from "Abstainer" to "Regular Heavy Drinker with Problems"). The absence of any association in males may be due to their inability to model binge drinking [75]; however, our results suggest otherwise. Additionally, the recent Clarke et al. [28] total weekly alcohol GWAS found sex-specific genetic correlation differences with an $r_{\mathrm{g}}=0.1$ in men and 0.33 in women. Taken together, our findings suggest EA may partially account for some of these observed gender gaps in alcohol consumption, but not others. We should note that the only available sex-specific EA GWAS had significant overlap $(\geq 18.9 \%)$ with the outcome datasets, so our exploratory sex-specific analysis used the same EA GWAS combining men and women. The lack of available sex-specific AD GWAS also meant we were unable to examine differences in AD risk. Notably, the sex-specific EA GWAS demonstrated nearly identical effect sizes between men and women, which support the validity of the estimates derived from the combined-sex EA GWAS, but future studies using sex-specific instruments are required.

\section{Strengths and limitations}

We note several strengths. We have analyzed multiple alcohol-related behavioral phenotypes, which support the consistency of our results. We have implemented multiple complementary MR methods (IVW, Egger, weighted median, and weighted mode MR) and diagnostics. Consistency of results across MR methods (accommodating different assumptions about genetic pleiotropy) strengthens our causal interpretation of the estimates [76]. We also used the largest publicly available GWASs for both exposure and outcome samples; large summary datasets are important for MR and other genetic analysis investigating small effect sizes [77]. We also note limitations and future directions. There is minimal sample overlap between the exposure SSGAC GWAS and the outcome PGC GWAS (AD), but there may still be individuals participating in multiple surveys, which event we cannot ascertain with available summary-level GWAS statistics. Further, the GWASs cohorts are from Anglophone countries, where beer is the preferred drink [78]; therefore, applicability to other countries with different alcohol preferences may be limited. Further still, it has been reported the UKB sample is more educated, with healthier lifestyles, and fewer health problems than the UK population [79], which may limit the generalizability to other populations. Replication of these findings using alcohol use information from different ethnicities is necessary. EA only measured years of completed schooling; determining how various aspects of education differentially impact alcohol consumption was not possible but should be a topic of future work. Finally, alcohol consumption is not stable over time [15]; however, the alcohol consumption outcomes correspond to current drinking behavior, which may have led to the misclassification of some individuals. The current drinking also impacts the temporal relationship of our bidirectional analyses, since the current alcohol intake likely occurred after maximum educational attainment for most of the participants. Future GWAS that evaluate drinking behavior during adolescence, or other longitudinal studies are necessary to confirm these findings and better elucidate the impact of alcohol intake on EA.

\section{Conclusions}

Our data show evidence of a causal relationship between EA and patterns of drinking behavior rather than overall total alcohol consumption highlighting that drinking metrics cannot necessarily be used interchangeably. Higher EA was linked with lower binge drinking, reduced total drinks on drinking days, more frequent drinking at meals, and use of moderate alcohol content beverages (such as wine). Additional education significantly reduced the risk of alcohol dependence. Alcohol consumption patterns may be significant pathways or mediators in the relationship between EA and health outcomes. In conjunction with the evidence demonstrating the causal role of education on other health behaviors, our findings suggest that increasing EA may be a 
useful target for prevention programs against problematic alcohol use and its consequences.

\section{Code availability}

The analysis code in $\mathrm{R}$ is available on request and all data displayed in the figures are available in the Supplementary Tables

Acknowledgements This research was facilitated by the Social Science Genetic Association Consortium (SSGAC), the Substance Use Disorders Working Group of the Psychiatric Genomics Consortium (PGC-SUD) (supported by funds from NIDA and NIMH to MH109532 and, previously, with analyst support from NIAAA to U01AA008401 (COGA)), and the Medical Research Council Integrative Epidemiology Unit (MRC-IEU, University of Bristol, UK), especially the developers of the MRC-IEU UKB GWAS Pipeline. We gratefully acknowledge their contributing studies and the participants in those studies without whom this effort would not be possible. This work was supported by the National Institutes of Health (NIH) intramural funding [ZIA-AA000242 to FWL]; Division of Intramural Clinical and Biological Research of the National Institute on Alcohol Abuse and Alcoholism (NIAAA). TKC, AMM, and MA are supported by the Wellcome Trust (Wellcome Trust Strategic Award "Stratifying Resilience and Depression Longitudinally" (STRADL) Reference 104036/Z/14/Z)

\section{Compliance with ethical standards}

Conflict of interest The authors declare that they have no conflict of interest.

Publisher's note Springer Nature remains neutral with regard to jurisdictional claims in published maps and institutional affiliations.

Open Access This article is licensed under a Creative Commons Attribution 4.0 International License, which permits use, sharing, adaptation, distribution and reproduction in any medium or format, as long as you give appropriate credit to the original author(s) and the source, provide a link to the Creative Commons license, and indicate if changes were made. The images or other third party material in this article are included in the article's Creative Commons license, unless indicated otherwise in a credit line to the material. If material is not included in the article's Creative Commons license and your intended use is not permitted by statutory regulation or exceeds the permitted use, you will need to obtain permission directly from the copyright holder. To view a copy of this license, visit http://creativecommons. org/licenses/by/4.0/.

\section{References}

1. Grant BF, Chou SP, Saha TD, Pickering RP, Kerridge BT, Ruan WJ, et al. Prevalence of 12-month alcohol use, high-risk drinking, and DSM-IV alcohol use disorder in the United States, 2001-2002 to 2012-2013: results from the national epidemiologic survey on alcohol and related conditions. JAMA Psychiat. 2017;74:911-23.

2. Griswold MG, Fullman N, Hawley C, Arian N, Zimsen SRM, Tymeson HD, et al. Alcohol use and burden for 195 countries and territories, 1990-2016: a systematic analysis for the Global Burden of Disease Study 2016. Lancet. 2018;392:1015-35.

3. Health Organization W. Global status report on alcohol and health. Geneva, Switzerland: WHO, 2014.
4. Rehm J, Mathers C, Popova S, Thavorncharoensap M, Teerawattananon Y, Patra J. Alcohol and Global Health 1 Global burden of disease and injury and economic cost attributable to alcohol use and alcohol-use disorders. Lancet. 2009;373:2223-33.

5. Corrao G, Bagnardi V, Zambon A, La Vecchia C. A meta-analysis of alcohol consumption and the risk of 15 diseases. Prev Med. 2004;38:613-9.

6. Praud D, Rota M, Rehm J, Shield K, Zatonski W, Hashibe M, et al. Cancer incidence and mortality attributable to alcohol consumption. Int J Cancer. 2016;138:1380-7.

7. Baliunas D, Rehm J, Irving H, Shuper P. Alcohol consumption and risk of incident human immunodeficiency virus infection: a meta-analysis. Int J Public Health. 2010;55:159-66.

8. Roerecke M, Rehm J. Chronic heavy drinking and ischaemic heart disease: a systematic review and meta-analysis. Open Heart. 2014;1:e000135.

9. Roerecke M, Rehm J. Irregular heavy drinking occasions and risk of ischemic heart disease: a systematic review and meta-analysis. Am J Epidemiol. 2010;171:633-44.

10. Piano MR, Phillips SA. Alcoholic cardiomyopathy: pathophysiologic insights. Cardiovasc Toxicol. 2014;14:291-308.

11. Kessler RC, Chiu WT, Demler O, Merikangas KR, Walters EE. Prevalence, severity, and comorbidity of 12-month DSM-IV disorders in the National Comorbidity Survey Replication. Arch Gen Psychiatry. 2005;62:617-27.

12. Arranz S, Chiva-Blanch G, Valderas-Martinez P, Medina-Remon A, Lamuela-Raventos RM, Estruch R. Wine, beer, alcohol and polyphenols on cardiovascular disease and cancer. Nutrients. 2012;4:759-81.

13. Collins SE. Associations between socioeconomic factors and alcohol outcomes. Alcohol Res: Curr Rev. 2016;38:83-94.

14. Kuntsche E, Knibbe R, Gmel G, Engels R. 'I drink spirits to get drunk and block out my problems...' beverage preference, drinking motives and alcohol use in adolescence. Alcohol Alcohol. 2006;41:566-73.

15. Marees AT, Smit DJA, Ong JS, MacGregor S, An J, Denys D, et al. Potential influence of socioeconomic status on genetic correlations between alcohol consumption measures and mental health. Psychol Med. 2019;1-15. https://doi.org/10.1017/ S0033291719000357. [Epub ahead of print].

16. Understanding the relationship between education and health: a review of the evidence and an examination of community perspective. https://www.ahrq.gov/professionals/education/curriculum-tools/ population-health/zimmerman.html, 2015, Accessed date 2015 .

17. National Center for Health S. Health, United States. Health, United States, 2011: with special feature on socioeconomic status and health. National Center for Health Statistics (US): Hyattsville (MD); 2012.

18. Grittner U, Kuntsche S, Gmel G, Bloomfield K. Alcohol consumption and social inequality at the individual and country levels-results from an international study. Eur J Public Health. 2013;23:332-9.

19. Katikireddi SV, Whitley E, Lewsey J, Gray L, Leyland AH. Socioeconomic status as an effect modifier of alcohol consumption and harm: analysis of linked cohort data. Lancet Public Health. 2017;2:e267-76.

20. Hingson RW, Zha W, White AM. Drinking beyond the binge threshold: predictors, consequences, and changes in the U.S. Am J Prev Med. 2017;52:717-27.

21. Balsa AI, Giuliano LM, French MT. The effects of alcohol use on academic achievement in high school. Econ Educ Rev. 2011;30:1-15.

22. Phillips AN, Smith GD. How Independent are independent effects-relative risk-estimation when correlated exposures are measured imprecisely. J Clin Epidemiol. 1991;44:1223-31. 
23. Smith GD, Ebrahim S. 'Mendelian randomization': can genetic epidemiology contribute to understanding environmental determinants of disease? Int J Epidemiol. 2003;32:1-22.

24. Smith GD. Use of genetic markers and gene-diet interactions for interrogating population-level causal influences of diet on health. Genes Nutr. 2011;6:27-43.

25. Saunders JB, Aasland OG, Babor TF, de la Fuente JR, Grant M. Development of the Alcohol Use Disorders Identification Test (AUDIT): WHO collaborative project on early detection of persons with harmful alcohol consumption-II. Addiction. 1993;88:791-804.

26. Sanchez-Roige S, Palmer AA, Fontanillas P, Elson SL, Adams MJ, Howard DM, et al. Genome-wide association study metaanalysis of the Alcohol Use Disorders Identification Test (AUDIT) in two population-based cohorts. Am J psychiatry. 2019;176:107-18.

27. Walters RK, Polimanti R, Johnson EC, McClintick JN, Adams MJ, Adkins AE, et al. Transancestral GWAS of alcohol dependence reveals common genetic underpinnings with psychiatric disorders. Nat Neurosci. 2018;21:1656.

28. Clarke TK, Adams MJ, Davies G, Howard DM, Hall LS, Padmanabhan S, et al. Genome-wide association study of alcohol consumption and genetic overlap with other health-related traits in UK Biobank ( $\mathrm{N}=112$ 117). Mol Psychiatry. 2017;22:1376-84.

29. Leamer EE. Lets take the con out of econometrics. Am Econ Rev. 1983;73:31-43.

30. Smith GD, Ebrahim S. Epidemiology-is it time to call it a day? Int J Epidemiol. 2001;30:1-11.

31. Davey Smith G, Hemani G. Mendelian randomization: genetic anchors for causal inference in epidemiological studies. Hum Mol Genet. 2014;23(R1):R89-98.

32. Bowden J, Smith GD, Haycock PC, Burgess S. Consistent estimation in Mendelian randomization with some invalid instruments using a weighted median estimator. Genet Epidemiol. 2016;40:304-14.

33. Hemani G, Zheng J, Elsworth B, Wade KH, Haberland V, Baird $\mathrm{D}$, et al. The MR-Base platform supports systematic causal inference across the human phenome. Elife. 2018;7.

34. Tillmann T, Vaucher J, Okbay A, Pikhart H, Peasey A, Kubinova $\mathrm{R}$, et al. Education and coronary heart disease: mendelian randomisation study. BMJ-Brit Med J. 2017;358:1-10.

35. Gage SH, Bowden J, Smith GD, Munafo MR. Investigating causality in associations between education and smoking: a twosample Mendelian randomization study. Int $\mathbf{J}$ Epidemiol. 2018;47:1131-40.

36. Okbay A, Beauchamp JP, Fontana MA, Lee JJ, Pers TH, Rietveld $\mathrm{CA}$, et al. Genome-wide association study identifies 74 loci associated with educational attainment. Nature. 2016;533:539-42.

37. Karlsson Linnér R, Biroli P, Kong E, Meddens SFW, Wedow R, Fontana MA, et al. Genome-wide association analyses of risk tolerance and risky behaviors in over 1 million individuals identify hundreds of loci and shared genetic influences. Nat Genet. 2019;51:245-57.

38. Elsworth B, Mitchell R, Raistrick CA, Paternoster L, Hemani G, Gaunt TR. MRC IEU UK Biobank GWAS pipeline version 1. Bristol, UK: University of Bristol; 2017.

39. Wilsnack RW, Wilsnack SC, Kristjanson AF, Vogeltanz-Holm ND, Gmel G. Gender and alcohol consumption: patterns from the multinational GENACIS project. Addiction. 2009; 104:1487-1500.

40. Morris TT, Davies NM, Davey Smith G. Can education be personalised using pupils' genetic data? bioRxiv. 2019: 645218 .

41. Millard LAC, Davies NM, Gaunt TR, Davey Smith G, Tilling K. Software Application Profile: PHESANT: a tool for performing automated phenome scans in UK Biobank. Int J Epidemiol.
2017;1-7. https://doi.org/10.1093/ije/dyx204. [Epub ahead of print]

42. Burgess S, Davies NM, Thompson SG. Bias due to participant overlap in two-sample Mendelian randomization. Genet Epidemiol. 2016;40:597-608.

43. Bulik-Sullivan BK, Loh P-R, Finucane HK, Ripke S, Yang J., Schizophrenia Working Group of the Psychiatric Genomics C, et al. LD score regression distinguishes confounding from polygenicity in genome-wide association studies. Nat Genet. 2015;47:291

44. Zheng J, Erzurumluoglu AM, Elsworth BL, Kemp JP, Howe L, Haycock PC, et al. LD Hub: a centralized database and web interface to perform LD score regression that maximizes the potential of summary level GWAS data for SNP heritability and genetic correlation analysis. Bioinformatics. 2017;33:272-9.

45. Bowden J, Smith GD, Burgess S. Mendelian randomization with invalid instruments: effect estimation and bias detection through Egger regression. Int J Epidemiol. 2015;44:512-25.

46. Bowden J, Del Greco MF, Minelli C, Smith GD, Sheehan N, Thompson J. A framework for the investigation of pleiotropy in two-sample summary data Mendelian randomization. Stat Med. 2017;36:1783-802.

47. Bowden J, Del Greco MF, Minelli C, Zhao Q, Lawlor DA, Sheehan NA, et al. Improving the accuracy of two-sample summary-data Mendelian randomization: moving beyond the NOME assumption. Int J Epidemiol. 2019;48:728-42.

48. Verbanck M, Chen CY, Neale B, Do R. Detection of widespread horizontal pleiotropy in causal relationships inferred from Mendelian randomization between complex traits and diseases (vol 50, 693, 2018). Nat Genet. 2018;50:1196-96.

49. Hemani G, Tilling K, Smith GD. Orienting the causal relationship between imprecisely measured traits using GWAS summary data. PLoS Genet. 2017;13:1-22.

50. Verbanck M, Chen C-Y, Neale B, Do R. Detection of widespread horizontal pleiotropy in causal relationships inferred from Mendelian randomization between complex traits and diseases. Nat Genet. 2018;50:693-8.

51. Auter Z. Beer reigns as Americans preferred alcoholic beverage. vol. 2019. Gallup; 2016.

52. Davies NM, Dickson M, Smith GD, van den Berg GJ, Windmeijer F. The causal effects of education on health outcomes in the UK Biobank. Nat Hum Behav. 2018;2:117-25.

53. Crum RM, Anthony JC. Educational level and risk for alcohol abuse and dependence: differences by race-ethnicity. Ethn Dis. 2000;10:39-52.

54. Kranzler HR, Zhou H, Kember RL, Vickers Smith R, Justice AC, Damrauer S, et al. Genome-wide association study of alcohol consumption and use disorder in 274,424 individuals from multiple populations. Nat Commun. 2019;10:1499.

55. Kranzler HR, Soyka M. Diagnosis and pharmacotherapy of alcohol use disorder: a review. JAMA. 2018;320:815-24.

56. Naimi TS, Brewer RD, Miller JW, Okoro C, Mehrotra C. What do binge drinkers drink? Implications for alcohol control policy. Am J Preventive Med. 2007;33:188-93.

57. Gronbaek M, Jensen MK, Johansen D, Sorensen TI, Becker U. Intake of beer, wine and spirits and risk of heavy drinking and alcoholic cirrhosis. Biol Res. 2004;37:195-200.

58. Lintonen TP, Konu AI. Adolescent alcohol beverage type choices reflect their substance use patterns and attitudes. J Youth Adolescence. 2003;32:279-89.

59. Siegel MB, Naimi TS, Cremeens JL, Nelson DE. Alcoholic beverage preferences and associated drinking patterns and risk behaviors among high school youth. Am J Prev Med. 2011;40:419-26.

60. Clarke TK, Smith AH, Gelernter J, Kranzler HR, Farrer LA, Hall LS, et al. Polygenic risk for alcohol dependence associates with 
alcohol consumption, cognitive function and social deprivation in a population-based cohort. Addict Biol. 2016;21:469-80.

61. Emberson JR, Bennett DA. Effect of alcohol on risk of coronary heart disease and stroke: causality, bias, or a bit of both? Vasc Health Risk Manag. 2006;2:239-49.

62. Dey M, Gmel G, Studer J, Dermota P, Mohler-Kuo M. Beverage preferences and associated drinking patterns, consequences and other substance use behaviours. Eur J Public Health. 2014; 24:496-501.

63. van Kippersluis H, O’Donnell O, van Doorslaer E. Long run returns to education: does schooling lead to an extended old age? J Hum Resour. 2009;4:1-33.

64. Naess O, Hoff DA, Lawlor D, Mortensen LH. Education and adult cause-specific mortality-examining the impact of family factors shared by 871367 Norwegian siblings. Int J Epidemiol. 2012;41:1683-91.

65. Lundborg P, Lyttkens CH, Nystedt P. The Effect of schooling on mortality: new evidence from 50,000 Swedish twins. Demography. 2016;53:1135-68.

66. Madsen M, Andersen PK, Gerster M, Andersen AMN, Christensen K, Osler M. Are the educational differences in incidence of cardiovascular disease explained by underlying familial factors? A twin study. Soc Sci Med. 2014;118:182-90.

67. Trevisan M, Dorn J, Falkner K, Russell M, Ram M, Muti P, et al. Drinking pattern and risk of non-fatal myocardial infarction: a population-based case-control study. Addiction. 2004;99:313-22.

68. Wood AM, Kaptoge S, Butterworth AS. Risk thresholds for alcohol consumption: combined analysis of individual-participant data for 599912 current drinkers in 83 prospective studies (vol 391, pg 1513, 2018). Lancet. 2018;391:2212-12.

69. Millwood IY, Walters RG, Mei XW, Guo Y, Yang L, Bian Z, et al. Conventional and genetic evidence on alcohol and vascular disease aetiology: a prospective study of 500000 men and women in China. Lancet. 2019;393:1831-42.
70. Maurage P, Pesenti M, Philippot P, Joassin F, Campanella S. Latent deleterious effects of binge drinking over a short period of time revealed only by electrophysiological measures. J Psychiatry Neurosci: JPN. 2009;34:111-8.

71. Piano MR, Mazzuco A, Kang M, Phillips SA. Cardiovascular consequences of binge drinking: an integrative review with implications for advocacy, policy, and research. Alcohol Clin Exp Res. 2017;41:487-96.

72. Carter AR, Gill D, Davies NM, Taylor AE, Tillmann T, Vaucher $\mathrm{J}$, et al. Understanding the consequences of education inequality on cardiovascular disease: mendelian randomisation study. BMJ (Clin Res ed). 2019;365:11855.

73. Belsky DW, Domingue BW, Wedow R, Arseneault L, Boardman JD, Caspi A, et al. Genetic analysis of social-class mobility in five longitudinal studies. Proc Natl Acad Sci. 2018;115:E7275.

74. Wood PK, Sher KJ, Erickson DJ, DeBord KA. Predicting academic problems in college from freshman alcohol involvement. $\mathrm{J}$ Stud Alcohol. 1997;58:200-10.

75. Huerta MC, Borgonovi F. Education, alcohol use and abuse among young adults in Britain. Soc Sci Med. 2010;71:143-51.

76. Lawlor DA. Commentary: Two-sample Mendelian randomization: opportunities and challenges. Int J Epidemiol. 2016;45:908-15.

77. Gage SH, Jones HJ, Burgess S, Bowden J, Smith GD, Zammit S, et al. Assessing causality in associations between cannabis use and schizophrenia risk: a two-sample Mendelian randomization study. Psychol Med. 2017;47:971-80.

78. Makela P, Gmel G, Grittner U, Kuendig H, Kuntsche S, Bloomfield $\mathrm{K}$, et al. Drinking patterns and their gender differences in Europe. Alcohol Alcohol (Oxf, Oxfs). 2006;41(Supplement): i8-18.

79. Fry A, Littlejohns TJ, Sudlow C, Doherty N, Adamska L, Sprosen $\mathrm{T}$, et al. Comparison of sociodemographic and health-related characteristics of UK biobank participants with those of the general population. Am J Epidemiol. 2017;186:1026-34. 